\title{
Aplicação da lógica fuzzy na análise sensorial de pão de forma enriquecido
}

\author{
Mônica T. Cavalcanti' ${ }^{1}$, Flávio L. H. da Silva ${ }^{2}$, José H. F. Cavalcanti², \\ Eliane R. Florentino ${ }^{3}$, Isanna M. Florêncio ${ }^{3}$ \& Ricardo T. Moreira $^{2}$
}

RESUMO

A lógica fuzzy vem sendo aplicada em diversas áreas do conhecimento e nela cada variável possui um conjunto de funções de pertinência representadas em um universo $X$ as quais geralmente exprimem o conjunto de variáveis linguísticas do sistema. Neste trabalho se propõe uma discussão sobre a utilização da lógica fuzzy na aceitação sensorial, a partir da opinião dos provadores quanto ao pão de forma enriquecido com amêndoas e proteínas de faveleira (Cnidosculus phyllacanthus (Mart.) Pax. et K. Hoffm.). Observou-se que o método fuzzy desenvolvido é de fácil utilização e o usuário do sistema só necessita encontrar a curva do centro de gravidade (CG) com maior valor final para indicar a amostra de maior aceitação, podendo utilizar as derivadas das curvas CG no auxílio da detecção da melhor escolha em algumas situações em que lancem dúvida, além de ser utilizado em conjunto com outro método estatístico.

Palavras-chave: faveleira, aceitação global, teste de aceitação

\section{Fuzzy logic application in sensory analysis of enriched loaf}

\begin{abstract}
Fuzzy logic is applied in many areas of knowledge. In this logic every variable has a set of functions, represented in an $X$ universe, which generally expresses a linguistic variables group of the system. This paper proposes a discussion on the use of fuzzy logic in sensory acceptance, from the taster's views of 'faveleira' (Cnidosculus phyllacanthus (Mart.)Pax. et K. Hoffm.) almonds and protein bread. It was observed that the developed controller method is easy to use and system's user only needs to find the curve of the GC with a final higher value to indicate the sample with greater acceptance. The user may use the derived CG curves aiding in the detection of the best choice in some situations, furthermore used in conjunction with other statistical method.
\end{abstract}

Key words: Cnidosculus phyllacanthus, global acceptation, acceptation test

1 UATA/UFCG. Rua Jairo Vieira Feitosa, s/n, Bairro dos Pereiros, CEP 58840-000, Pombal, PB. Fone: (83) 3431-4000. E-mail: monicatejoc@yahoo.com.br ${ }^{2}$ CT/UFPB, CEP 58051-900, Cidade Universitária, João Pessoa, PB. Fone: (83) 9921-9209.. E-mail: flavioluizh@yahoo.com.br; zevhom@uol.com.br; ricardo.ufpb@gmail.com

${ }^{3}$ CCT/UEPB. Av. das Baraúnas, s/n, Bodocongó, Campina Grande, PB. Fone: (83) 8881-9340. E-mail: elianerf@yahoo.com.br; isannamf@yahoo.com.br 


\section{INTRODUÇÃO}

A análise sensorial é uma ciência interdisciplinar através na qual se convidam avaliadores que se utilizam da complexa interação dos sentidos para medir ou avaliar as características sensoriais e a aceitabilidade dos produtos alimentícios e muitos outros materiais (Battochio et al., 2006; Azevedo et al., 2011).

Os métodos sensoriais são baseados nas respostas aos estímulos haja vista que produzem sensações cujas dimensões são: intensidade, extensão, duração, qualidade e prazer ou desprazer; enquanto os estímulos podem ser medidos por métodos físicos e químicos, as sensações são medidas por processos psicológicos.

Uma das exigências da avaliação sensorial é a decisão sobre a qualidade do produto em que nos testes de escala hedônica é traduzida pela aceitação dos alimentos. Existem alguns métodos estatísticos desenvolvidos para a análise sensorial, como a análise de variância, análise de superfície de resposta e redes neurais artificiais, entre outros.

A lógica fuzzy foi proposta por Zadeh, na década de 1960, e está sendo aplicada em diversas áreas do conhecimento (Liu et al., 2006; Nolasco-Carvalho et al., 2009; Oliveira \& Montini, 2009; Rodrigues Júnior et al., 2011). Na lógica fuzzy a qualitatividade é a propriedade de um produto possuir qualidade em circunstâncias de ambiguidade. Esta propriedade se adere às condições de apreciação dos produtos alimentícios possibilitando a utilização de uma forma quantitativa alternativa. Atributos devem fornecer descrições vinculadas às características humanas e àquelas sensoriais do produto que, através de modelo multiatributo mapeado pelas funções de pertinência, converge à decisão otimizada. Na prática, a lógica fuzzy permite computar com palavras convertendo os estímulos em respostas ou medidas em ações de controle (Lanzillotti \& Lanzillotti, 1999).

Tradicionalmente, na lógica fuzzy uma das técnicas usadas na análise de dados é o cluster (agrupamento) que pode ser empregado na análise sensorial. No projeto do controlador fuzzy é requerida a fixação das variáveis da premissa e a definição das funções de pertinência ao conjunto fuzzy é usada no controlador (Kosko, 1996).

A faveleira (Cnidosculus phyllacanthus (Mart.) Pax. et K. Hoffm.) é uma planta encontrada em todos os estados do Nordeste brasileiro, principalmente nas regiões semiáridas do sertão e caatinga; possui sementes oleaginosas com alto teor de ácidos graxos essenciais, sobretudo o ômega-6, além de rica em proteínas de ótima qualidade com ausência de elementos tóxicos, razão pela qual está sendo estudada como alternativa alimentar (Cavalcanti \& Bora, 2010; Cavalcanti et al., 2011a,b).

Este trabalho propõe uma discussão sobre a utilização da lógica fuzzy como ferramenta para avaliar a aceitação sensorial a partir da opinião dos provadores quanto ao pão de forma enriquecido com sementes de faveleira.

\section{Material e Métodos}

As sementes da faveleira (Cnidoscolus phyllacanthus (Mart.) Pax et K. Hoffm.) utilizadas foram colhidas no município de Santa Luzia, distante 160 km de João Pessoa, estado da Paraíba, Brasil. As sementes foram separadas de contaminantes e impurezas, lavadas em água corrente e secadas em estufa de ar circulante (marca Nova Ética) a $40{ }^{\circ} \mathrm{C}$, durante $24 \mathrm{~h}$, trituradas e tamisadas para obtenção da farinha. $\mathrm{O}$ isolado protéico foi obtido segundo metodologia descrita por Cavalcanti et al. $(2009 ; 2011 b)$.

Obteve-se o pão de forma convencional a partir de uma formulação que utiliza a farinha de trigo como base visando à determinação das porcentagens dos demais ingredientes adicionados à massa (açúcar, gordura, sal, água e fermento); esta formulação foi modificada pela adição da farinha e proteína da faveleira, variáveis de entrada, de acordo com o planejamento experimental fatorial $2^{2}$ para avaliar quantitativamente a influência sobre a resposta (aceitação global do produto cujos níveis das variáveis independentes utilizadas em ordem crescente $(-1,0,+1)$ foram $2,5,5$ e $7,5 \%$ para a farinha e de $0,01,0,015$ e $0,02 \%$ para as proteínas da faveleira totalizando 7 experimentos. Na Tabela 1 estão expostos os números de experimentos realizados.

Tabela 1. Matriz do planejamento fatorial $2^{2}+3$ ponto central

\begin{tabular}{lcc}
\hline \multirow{2}{*}{ Experimentos } & \multicolumn{2}{c}{ Variáveis (faveleira) } \\
\cline { 2 - 3 } (Amostra 1) Padrão & Farinha (\%) & Proteínas (\%) \\
(Amostra 2) 1 & - & - \\
(Amostra 3) 2 & $(-1) 2,5$ & $(-1) 0,01$ \\
(Amostra 4) 3 & $(+1) 7,5$ & $(-1) 0,01$ \\
(Amostra 5) 4 & $(-1) 2,5$ & $(+1) 0,02$ \\
(Amostra 6) 5 & $(+1) 7,5$ & $(+1) 0,02$ \\
(Amostra 7) 6 & $(0) 5,0$ & $(0) 0,015$ \\
(Amostra 8) 7 & $(0) 5,0$ & $(0) 0,015$ \\
\hline
\end{tabular}

As amostras foram submetidas ao teste de aceitação global por escala hedônica utilizando-se metodologia recomendada pelo Instituto Adolfo Lutz (Brasil, 2005). Este teste foi conduzido com 50 provadores não-treinados, de ambos os sexos, representando o público consumidor do produto, através de uma escala hedônica de 9 pontos, com escores variando de 9 (gostei muitíssimo) até 1 (desgostei muitíssimo).

Devidamente codificadas as amostras foram apresentadas, aleatoriamente, aos provadores, em cabines individuais, servidas em pratos de plástico e de um copo de água mineral em temperatura ambiente. $O$ procedimento foi efetuado em sala com luz branca, equivalente à luz do dia. A sessão foi conduzida apresentando-se quatro amostras de pão correspondentes às formulações diferentes e, em seguida, mais quatro formulações, de acordo com o planejamento.

O Comitê de Ética da Universidade Estadual da Paraíba, localizada em Campina Grande, PB, aprovou o estudo em 23 de fevereiro de 2010, pelo processo CAAE - 0621.0.133.000-09.

Foi desenvolvido um programa denominado sistema sensorial fuzzy em que as respostas dos provadores foram analisadas através da lógica fuzzy e de um programa desenvolvido e escrito no ambiente Matlab ${ }^{\circledR}$ (MATrixLABoratory) software versão 7.0.

O procedimento para verificar a aplicabilidade da lógica fuzzy com vista à tomada de decisão na aceitabilidade de um produto em provas hedônicas na análise sensorial, foi conduzido a partir das seguintes hipóteses: 1) A lógica fuzzy pode ser aplicada para tomada de decisão na aceitabilidade 
de um produto submetido a provas hedônicas na análise sensorial; 2) As composições "característica do consumidor" e "sensações hedônicas provocadas pelo produto" compostas por fatores intervenientes, são os entes necessários à convergência ao conjunto decisório; 3) Existe uma hierarquização dos componentes intracomposição; 4) O nível de acuidade avaliativa da composição "característica do consumidor" na aceitabilidade do produto é diferenciado pelo atributo gênero e idade.

A "fuzzyficação" avaliou o grau de pertinência da entrada numérica fornecida $(\mathrm{X})$ ocorrendo uma ativação das regras fuzzy formando um conjunto de saída, que foi "defuzzyficado" para gerar um resultado numérico de saída conforme sugerido por Gomide \& Gudwin (1995).

\section{Resultados e Discussão}

O sistema sensorial fuzzy proposto neste trabalho foi baseado em controladores e requer, usualmente, um controle na forma de quantidade real e não na forma fuzzy enquanto o controlador fuzzy de Mamdani utiliza dois dispositivos para transformar uma variável da forma real para a forma fuzzy e vice-versa. Esses dispositivos são o fuzzyficador ("fuzzyfier") que precede o controlador fuzzy para associar um valor linguístico a uma quantidade real; já o "defuzzyficador" ("defuzzyfier") segue o controlador fuzzy, associa um número real a um conjunto (Mamdani \& Gaines, 1981; Cavalcanti et al., 2011a).

Zadeh (1978) afirma que cada variável fuzzy possui um conjunto de funções de pertinência $(\mu \mathrm{i}(\mathrm{X}))$, representadas em um universo $\mathrm{X}$ que, em geral, exprimem o conjunto de variáveis linguísticas do sistema em que cada função de pertinência possui uma variável lógica associada como verificado nesse trabalho.

Na Figura 1 observa-se a entrada $X$ de um sistema fuzzy que é aplicado sobre três funções de pertinência $Z(X), P(X), M(X)$ e $G(X)$ numa forma agrupada e essas funções de pertinência representam variáveis linguísticas fuzzy.

No centro da Figura 1 apresentou-se o agrupamento das funções de pertinência junto a uma função não linear, obtida da "defuzzyficação" (variável $\mathrm{CG}(\mathrm{X})$ na cor preta) usando-se o centro de gravidade. As operações feitas, inicialmente, entre

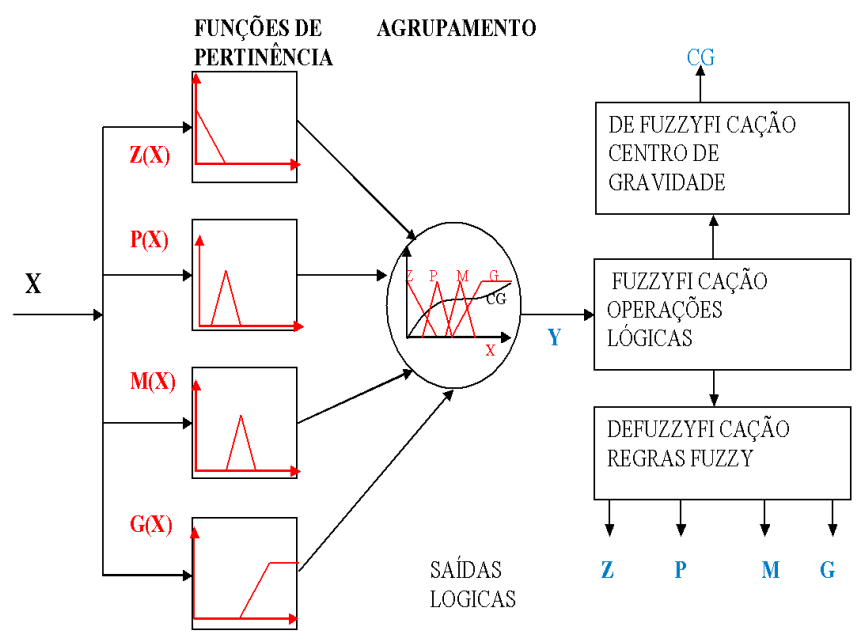

Figura 1. Diagrama geral de um sistema fuzzy as funções de pertinência da variável fuzzy e nos resultados obtidos $(\mathrm{Y})$ foram aplicadas as variáveis lógicas e discretas associadas a cada função de pertinência.

$\mathrm{Na}$ Figura 2 apresentam-se as curvas das funções de pertinência $(\mu \mathrm{i}(\mathrm{X}))$ e a curva da "defuzzyficação".

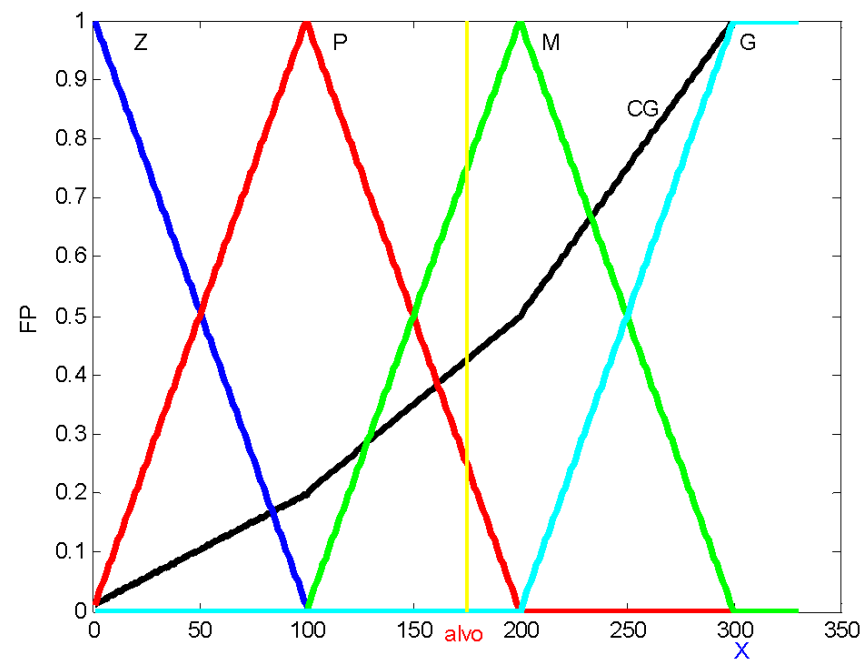

Figura 2. Funções de pertinência (FP) e curva CG

A abscissa das curvas da Figura 2 representa o universo de discurso da variável X a ser "fuzzyficada" $(0<X<330)$. As curvas das funções de pertinência estão desenhadas em diferentes cores em que a função de pertinência $Z(x)$ desenhada na cor azul escuro, representa a variável linguística $Z$ (zero) que, por sua vez, representa valor da variável $X$ próximo de zero, a função de pertinência $\mathrm{P}(\mathrm{x})$ é desenhada na cor vermelha, representando a variável linguística $\mathrm{P}$ (pequeno) que representa valor da variável $X$ pequeno, a função de pertinência $M(x)$ é desenhada na cor verde, representando a variável linguística $\mathrm{M}$ (médio) que representa valor da variável $\mathrm{X}$ médio em que a função de pertinência $G(x)$ é desenhada na cor azul claro e representa a variável linguística $\mathrm{G}$ (grande) que representa valor da variável $\mathrm{X}$ grande.

No centro da Figura 2 está desenhada uma reta amarela indicando a abscissa $X$ da variável alvo. Na abscissa indicada pelo alvo (alvo $=X=175$ ) a reta perpendicular à abscissa corta as curvas $\mathrm{P}(\mathrm{x})$ e $\mathrm{M}(\mathrm{x})$ nas ordenadas 0,25 e 0,75 , respectivamente; neste ponto $\mathrm{M}(\mathrm{x})$ tem a maior ordenada $(\mathrm{M}($ alvo $)>\mathrm{P}($ alvo $))$. Na fase de "defuzzyficação" considerou-se que $M(x)$ é a função de pertinência vencedora. Pode-se ver uma função não linear obtida da "defuzzyficação" (função CG(X)) usando-se o método do centro de gravidade.

No cálculo da função $\mathrm{CG}(\mathrm{X})$ através do método do centro de gravidade, necessitou-se da definição de pesos $\left(\mathrm{w}_{\mathrm{i}}\right)$ para as funções de pertinência $(Z(X), P(X), M(X)$ e $G(X))$. Definiu-se os valores crescentes para os pesos das funções de pertinência: ${ }_{w} 1=0,01 ;{ }_{w} 2=0,2 ;{ }_{w} 3=0,5 \mathrm{e}_{w} 4=1\left({ }_{w} 1\right.$ - peso de $Z(X),{ }_{w} 2$ - peso de $P(X)$, 3 - peso de $M(X) e_{w} 4$ - peso de $G(X)$ ). Fazendo-se a variável alvo variar de 0 até $\mathrm{o}$ valor máximo $(0<$ alvo $<330)$ usando-se a Eq. 1 para calcular o centro de gravidade CG (alvo) obtém-se a curva $\mathrm{CG}(\mathrm{X})$. 
A.

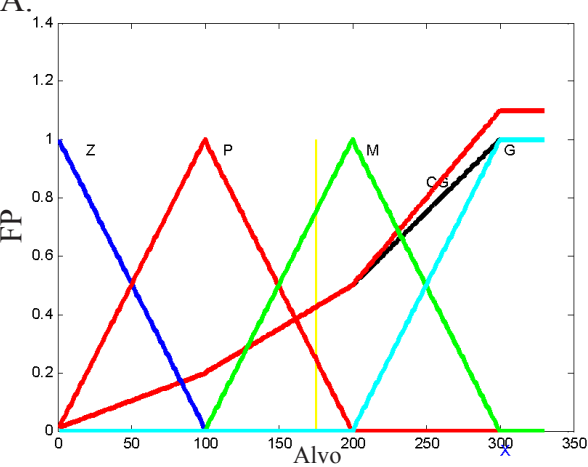

B.

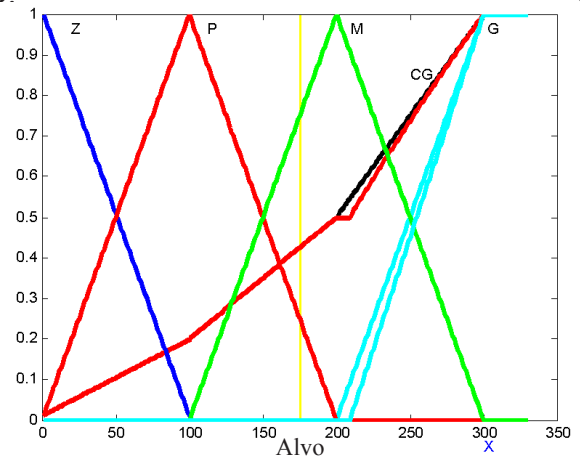

C.

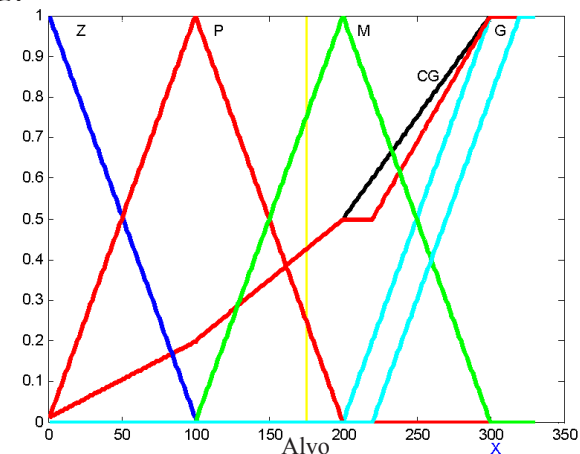

Figura 3. Sistema fuzzy, mudança no posicionamento (peso, declividade e posição)

$$
C G(x)=\frac{\sum_{i=1}^{k} \mu_{i}(x) \times w_{i}}{\sum_{i=1}^{k} \mu_{i}(x)}
$$

O cálculo do valor de $\mathrm{CG}(\mathrm{X})$ para $\mathrm{X}$ - alvo é feito com base na Eq. 1 na forma mostrada nas Eqs. 2 e 3.

$$
\begin{gathered}
\mathrm{CG}(\mathrm{x})=(\mathrm{Z}(\mathrm{X}) * \mathrm{wZ}+\mathrm{P}(\mathrm{X}) * \mathrm{wP}+\mathrm{M}(\mathrm{X}) * \mathrm{wM}+ \\
+\mathrm{G}(\mathrm{X}) * \mathrm{wG}) /(\mathrm{Z}(\mathrm{X})+\mathrm{P}(\mathrm{X})+\mathrm{M}(\mathrm{X})+\mathrm{G}(\mathrm{X})) \\
\mathrm{CG}(\mathrm{alvo})=\left(0 * 0,01+0,25 * 0,2+0,75^{*} 0,5+0 * 1\right) / \\
(0,0+0,25+0 ., 5+0,0)=0,425
\end{gathered}
$$

As características sensoriais das amostras podem ser descritas por adjetivos do tipo bom, ruim, entre outros; portanto, uma única característica da mistura pode ser definida por um conjunto de adjetivos, o que leva à utilização de histogramas centrados nos adjetivos e com intensidade igual ao número de escolhas dos adjetivos pelos provador e sabendo-se que a análise sensorial é feita a partir de opiniões de certo número de avaliadores (aval) para determinado número de amostras (am).

Sabendo-se que a opinião dos avaliadores pode ser descrita por adjetivos e que esses adjetivos podem ser representados numericamente como, por exemplo, um sistema fuzzy com quatro funções de pertinência: ruim $=0,1$; fraco $=0,3$; médio $=0,5$; bom $=0,7$ e ótimo $=1$ pode representar a opinião dos avaliadores. Sabendo-se que existem diferentes amostras a serem testadas pelos avaliadores e que essas amostras podem ser representadas na forma de histograma, analisou-se o sistema fuzzy para detectar o parâmetro a ser modificado. A curva $\mathrm{CG}(\mathrm{X})$ foi traçada com os valores dos posicionamentos mostrados na Eq. 4, pesos mostrados na Eq. 5 e declividade mostrada na Eq. 6.

$$
\begin{aligned}
& \operatorname{donde}(1)=1 ; \operatorname{donde}(2)=100 ; \\
& \operatorname{donde}(3)=200 ; \operatorname{donde}(4)=300 \\
& \operatorname{peso}(1)=0,01 ; \operatorname{peso}(2)=0,2 ; \\
& \operatorname{peso}(3)=0,5 ; \operatorname{peso}(4)=1 \\
& \operatorname{decliv}(1)=1 ; \operatorname{decliv}(2)=1 ; \\
& \operatorname{decliv}(3)=1 ; \operatorname{decliv}(4)=1
\end{aligned}
$$

Na Figura 3 apresentam-se três figuras do sistema fuzzy com a modificação (aumento do valor) nos parâmetros das funções de pertinência. À esquerda da parte superior da Figura 3 apresenta-se a curva CG obtida para mudança somente no peso da $\mu_{4}$, peso(4) $=1,1$; à direita da parte superior da Figura 3 apresenta-se a curva CG obtida para mudança só na inclinação da $\mu_{4}$, declive $(4)=1,1$ e na parte inferior da Figura 3 apresenta-se a curva CG obtida para mudança apenas no posicionamento da $\mu_{4}$, sendo $(4)=320$.

Observando as curvas das CG(X) apresentadas na Figura 3, pode-se notar que a variação da curva de $\mathrm{CG}(\mathrm{X})$ é mais suave quando se modifica o peso da função de pertinência $\left(\mu_{\mathrm{i}}\right)$; além disto e considerando pesos, declividade e posicionamento iguais, modificando-se o peso, a declividade ou o posicionamento das funções de pertinência, pode-se observar que é maior o valor final de $\mathrm{CG}(\mathrm{X})$ com o peso maior.

A partir dessas observações decidiu-se modificar os pesos das funções de pertinência com base no histograma das amostras. As decisões a serem tomadas através da lógica fuzzy sobre a característica da mistura foram feitas usando-se um histograma referente à opinião dos provadores, acoplado a curva do centro de gravidade das funções de pertinência do sistema usando-se a lógica fuzzy.
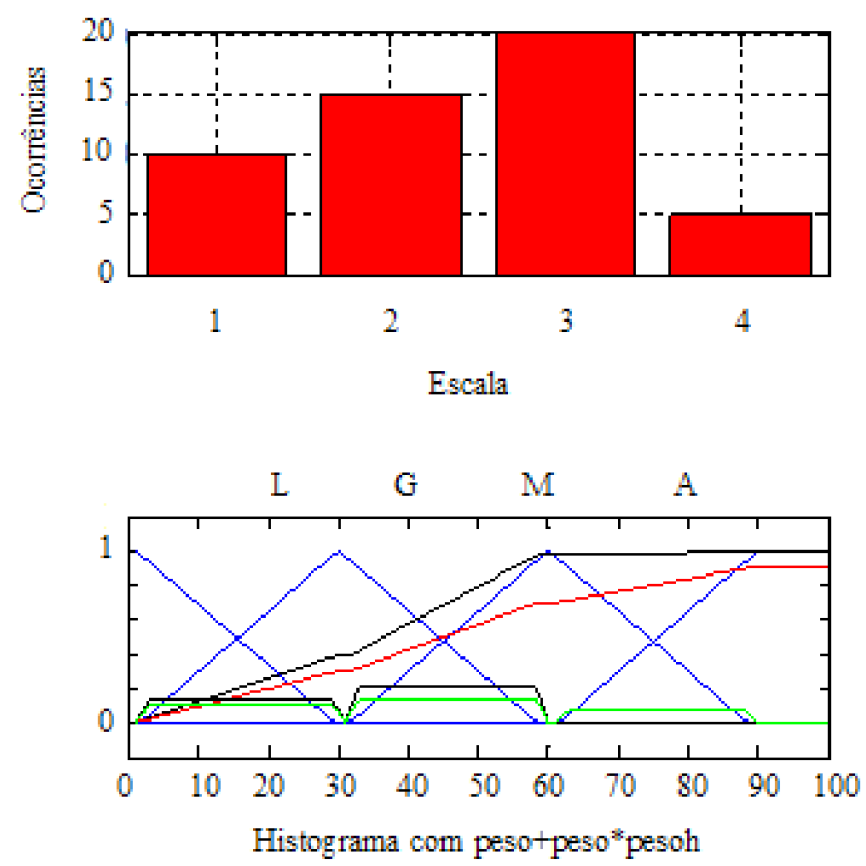

Figura 4. Histograma e função de pertinência 
No projeto do sistema sensorial fuzzy usou-se um conjunto de amostras hipotético para o projeto e análise do novo método ao qual se atribuiu uma variável fuzzy $\left(\mathrm{S}_{\mathrm{f}}\right)$ para representar a escolha sensorial da amostra e se associaram diferentes valores linguísticos para $\mathrm{S}_{\mathrm{f}}$ seguindo-se as variáveis linguísticas (gostei ligeiramente (L), gostei (G), gostei muito (M) e gostei muitíssimo (A)).

$\mathrm{Na}$ Figura 4 apresentam-se as curvas obtidas na análise fuzzy do conjunto de dados de uma amostra hipotética com um
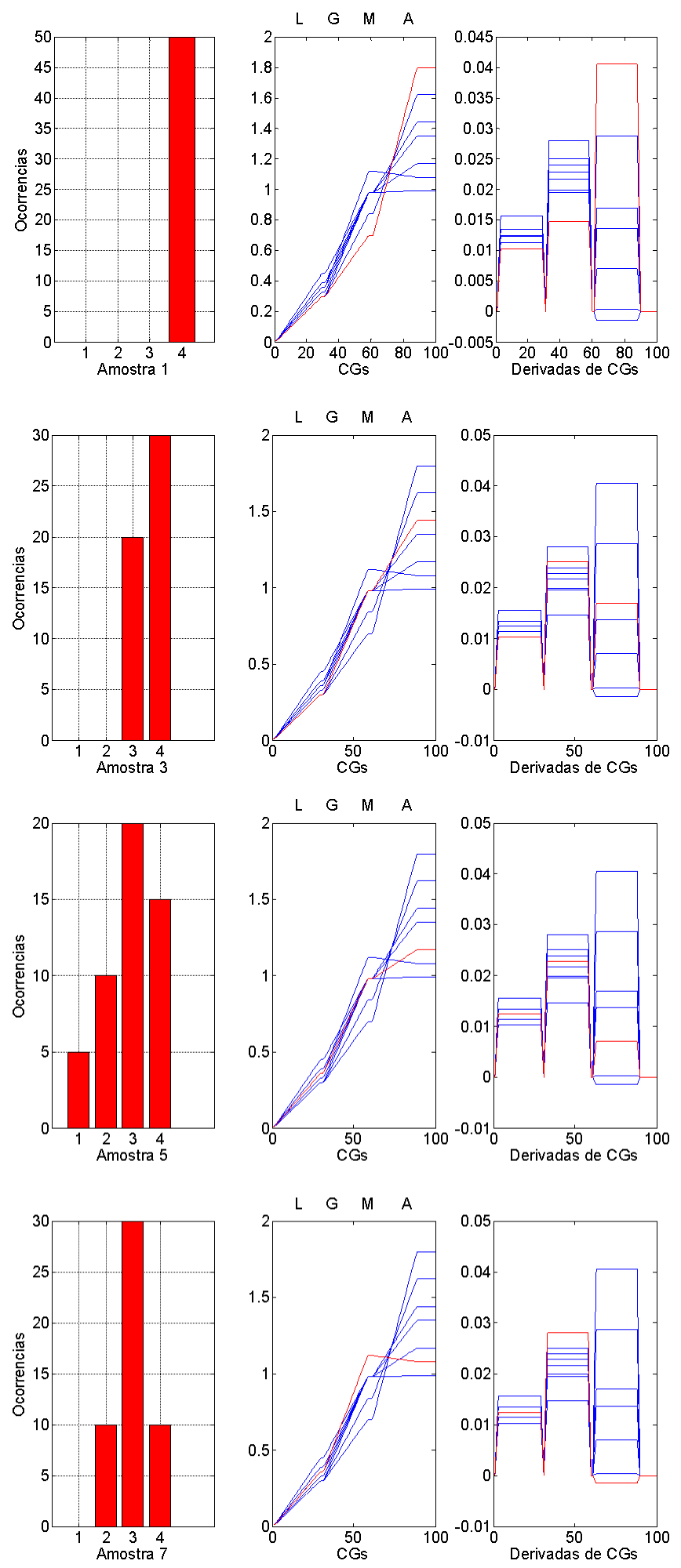

histograma obtido da análise dos provadores de uma amostra de uma mistura hipotética provada por cinquenta avaliadores representados pelo símbolo "elem $=50$ ". A abscissa representa os adjetivos escolhidos para classificarem as misturas e a altura das barras do histograma indica o número de provadores que escolheram aquele adjetivo. A abordagem foi acoplar o histograma à curva CG gerada com a Eq. 1; por exemplo, em um histograma com elem $=50$ provadores calculou-se um peso
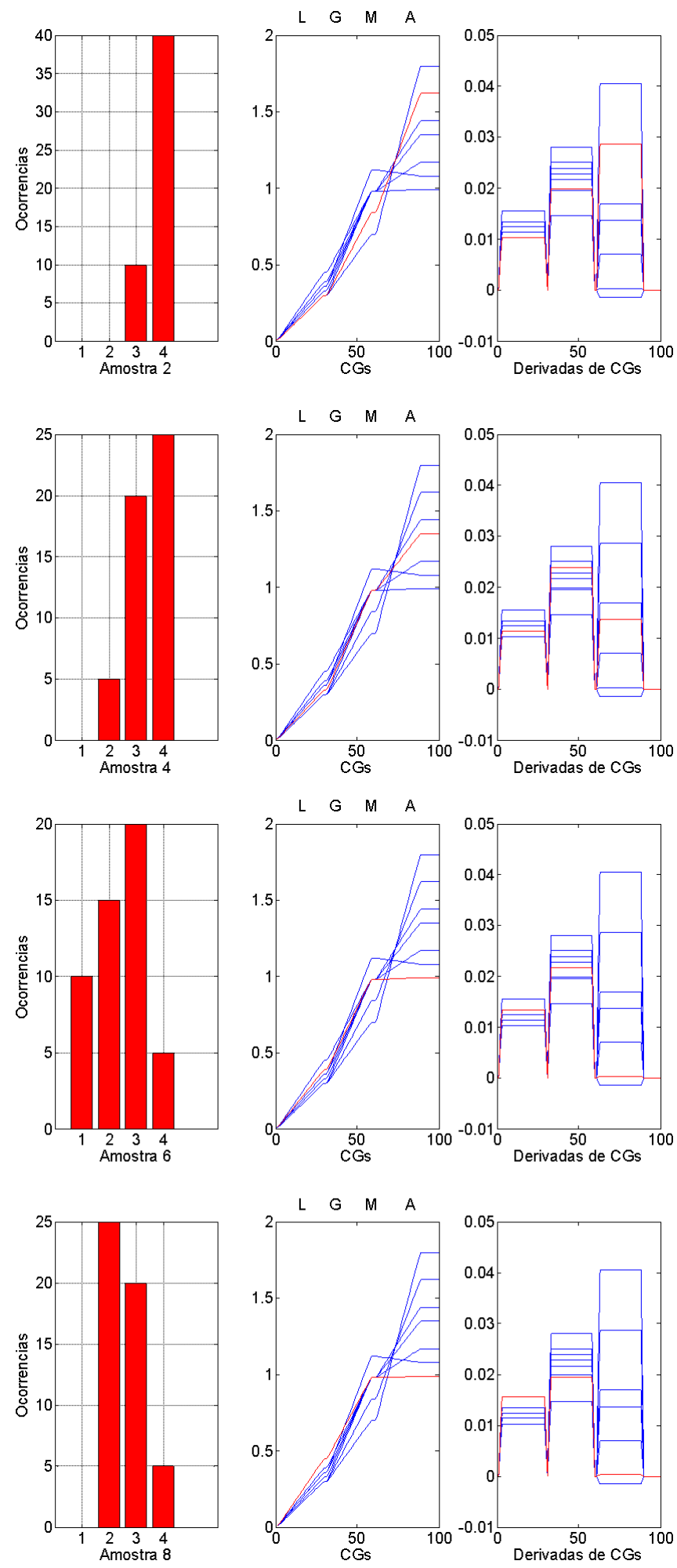

Figura 5. Histograma, curva de gravidade e derivadas de amostras hipotéticas na avaliação sensorial fuzzy 
auxiliar para as $\mu_{\mathrm{i}}$ "pesoh=hist/elem" que deve ser adicionado a cada peso das funções de pertinência $\left(\mu_{\mathrm{i}}=\mu_{\mathrm{i}}+\right.$ pesoh $\left._{\mathrm{i}}\right)$.

Na Figura 4 apresentam-se as funções de pertinência e a curva $\mathrm{CG}(\mathrm{X})$ verificando-se um conjunto de adjetivos que representam a opinião da amostra dos avaliadores. Observe-se que $\mathrm{CG}(\mathrm{X})$ tem forma aproximada de uma envoltória da curva dos histogramas. Na mesma figura foi desenhada a derivada de $C G(X)(d C G(X) / d X)$. A curva da derivada apresenta descontinuidade nos pontos entre as funções de pertinência e o seu valor é proporcional ao número do histograma da amostra. Essas características observadas foram usadas na análise sensorial das amostras usando-se a lógica fuzzy.

Na Figura 5 apresenta-se um resultado da análise fuzzy de oito amostras definidas empiricamente; as amostras são numeradas da esquerda para a direita e da parte superior para a inferior.

As amostras 1, 2, 3, 4, 5, 6, 7 e 8, com seus valores finais da curva $\mathrm{CG}(\mathrm{X})$ foram de, respectivamente, 1,80, 1,62, 1,44, 1,35, $1,17,0,99,1,08$ e 0,99 . Observa-se que o valor final de $\mathrm{CG}(\mathrm{X}) \mathrm{da}$ amostra $1(1,80)$ é maior que o valor final das demais amostras e após estudos comparativos com os métodos estatísticos tradicionais pode-se confirmar que a amostra indicada é aquela com a maior $\mathrm{CG}(\mathrm{X})$ sendo, portanto, a melhor avaliada pelos provadores; também se observa que o valor final de $\mathrm{CG}(\mathrm{X})$ da amostra $6(0,99)$ é igual ao valor final da amostra $8(0,99)$ e o desempate é feito analisando-se os valores da curva $\mathrm{dCG}(\mathrm{X}) / \mathrm{dX}$.

A partir da avaliação sensorial do pão de forma enriquecido com as sementes da faveleira montou-se um histograma das amostras em que um vetor variável de comprimento nove conta as ocorrências em cada gradação da avaliação e armazena os resultados no vetor histograma (vetor hist) que é uma matriz montada no programa. A cada gradação foi associada uma função da lógica fuzzy (função de pertinência, FP) que varia de gostei muitíssimo (armazenado numa tabela e atribuído o valor 9), a desgostei muitíssimo (armazenado numa tabela e atribuído o valor 1$)$.

A Tabela 2 apresenta as notas dos provadores sobre o conjunto das oito amostras de pão de forma enriquecido. Analisando esta tabela, vê-se que a distribuição das análises dos provadores pode ser analisada usando-se um histograma das amostras em relação à sua análise sensorial.

Para se conhecer a melhor interação na composição do pão entre a farinha e a proteína da faveleira utilizou-se uma associação entre o gosto dos provadores através da escala hedônica verbal e as variáveis linguísticas utilizadas na lógica fuzzy, em que a cada gradação foi associada uma função de pertinência que varia de gostei muitíssimo (armazenado e atribuído o valor 9), a desgostei muitíssimo (armazenado e atribuído o valor 1$)$.

Na Figura 6 apresentam-se as curvas obtidas na análise fuzzy do conjunto de dados da amostra 1.
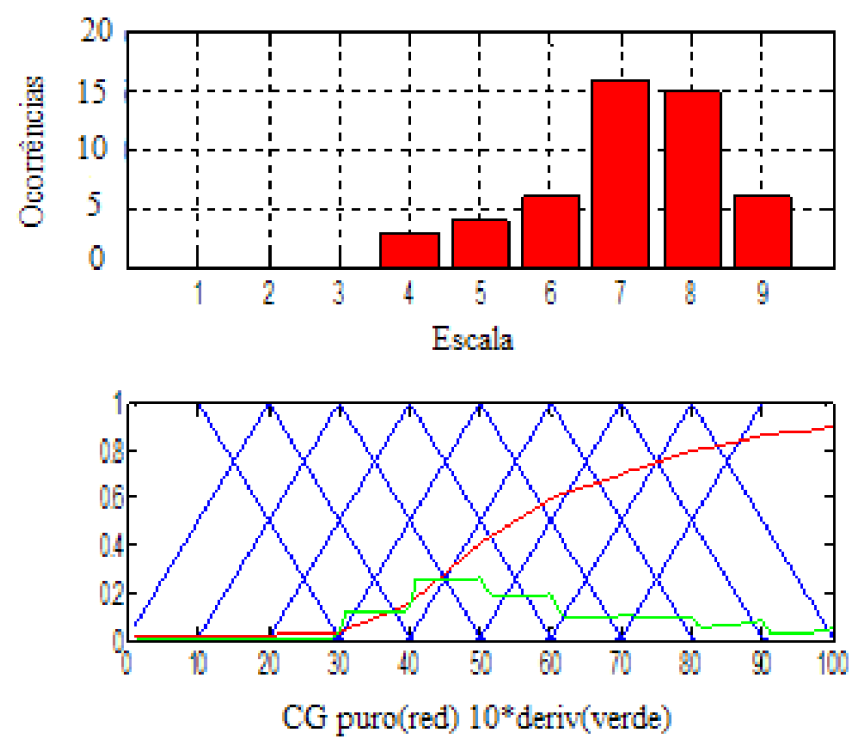

Figura 6. Histograma e análise fuzzy de $S_{f}$ com as funções de pertinência da amostra padrão (amostra 1)

As curvas das funções de pertinência da variável fuzzy $\mathrm{S}_{\mathrm{f}}$ estão representadas na cor azul e a escolha sensorial das amostras na cor amarela. Na Figura 6 apresentam-se a curva CG obtida através da Eq. 1 (cor vermelha) e a derivada da curva CG na cor verde; na Eq. 1 a FP representa o valor das FPs (0 $\left.\leq \mathrm{FP}_{\mathrm{i}} \leq 1\right)$ e $\mathrm{w}_{\mathrm{i}}$ representa o peso das FPs; por exemplo, a FP tem seu máximo em $\mathrm{x}=10$ (abscissa), possui uma inclinação de 6.6 e um peso igual a $\mathrm{w}_{1}=0.1$. Observe-se que a curva $\mathrm{CG}$ não depende do histograma, mas depende dos vetores.

Analisando o histograma da amostra 1 da Figura 6, tem-se

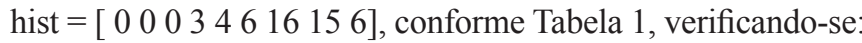
zero ocorrência de avaliações dos pontos 1, 2 e 3 (variáveis linguísticas:desgostei muitíssimo, desgostei muito e desgostei moderadamente; três ocorrências de avaliação do ponto 4 (desgostei ligeiramente); quatro ocorrências de avaliação do ponto 5 (Nem gostei/nem desgostei), seis do ponto 6 (gostei ligeiramente), dezesseis do ponto 7 (Gostei moderadamente), quinze do ponto 8 (gostei muito) e seis do ponto 9 (gostei

Tabela 2. Distribuição das notas atribuídas pelos provadores a cada amostra na analise sensorial

\begin{tabular}{|c|c|c|c|c|c|c|c|c|c|}
\hline \multirow{2}{*}{ Escala Hedônica } & \multirow{2}{*}{ Nota } & \multicolumn{8}{|c|}{ Experimentos/n provadores por nota } \\
\hline & & Padrão & 1 & 2 & 3 & 4 & 5 & 6 & 7 \\
\hline 1 - Desgostei muitíssimo & 1 & 0 & 0 & 0 & 0 & 1 & 0 & 0 & 0 \\
\hline 2 - Desgostei muito & 2 & 0 & 0 & 2 & 1 & 0 & 1 & 2 & 0 \\
\hline 3 - Desgostei moderadamente & 3 & 0 & 0 & 1 & 0 & 1 & 1 & 2 & 0 \\
\hline 4 - Desgostei ligeiramente & 4 & 3 & 0 & 3 & 1 & 4 & 5 & 3 & 1 \\
\hline 5 - Nem gostei/Nem desgostei & 5 & 4 & 4 & 5 & 5 & 5 & 2 & 1 & 4 \\
\hline 6 - Gostei ligeiramente & 6 & 6 & 10 & 10 & 11 & 9 & 10 & 9 & 6 \\
\hline 7 - Gostei moderadamente & 7 & 16 & 9 & 15 & 11 & 15 & 12 & 14 & 9 \\
\hline 8 - Gostei muito & 8 & 15 & 23 & 10 & 16 & 10 & 15 & 11 & 14 \\
\hline 9 - Gostei muitíssimo & 9 & 6 & 4 & 4 & 5 & 5 & 4 & 8 & 16 \\
\hline Média & & 7,08 & 7,26 & 6,50 & 6,94 & 6,58 & 6,70 & 6,78 & 7,30 \\
\hline Desvio padrão & & $\pm 1,34$ & $\pm 1,12$ & $\pm 1,67$ & $\pm 1,43$ & $\pm 1,68$ & $\pm 1,63$ & $\pm 1,82$ & $\pm 1,25$ \\
\hline
\end{tabular}


muitíssimo). A mesma análise foi feita para os demais histogramas das amostras.

A abscissa das FPs foi projetada para 100 pontos (variando de 1 a 100). Os máximos das FPs foram projetados para ocorrerem nos pontos 10, 20, 30, 40, 50, 60, 7080 e 90 da abscissa, para as escolhas (i) de 1 a 9, respectivamente. A declividade (decliv) das retas das FPs foi fixa e igual a 6.6.
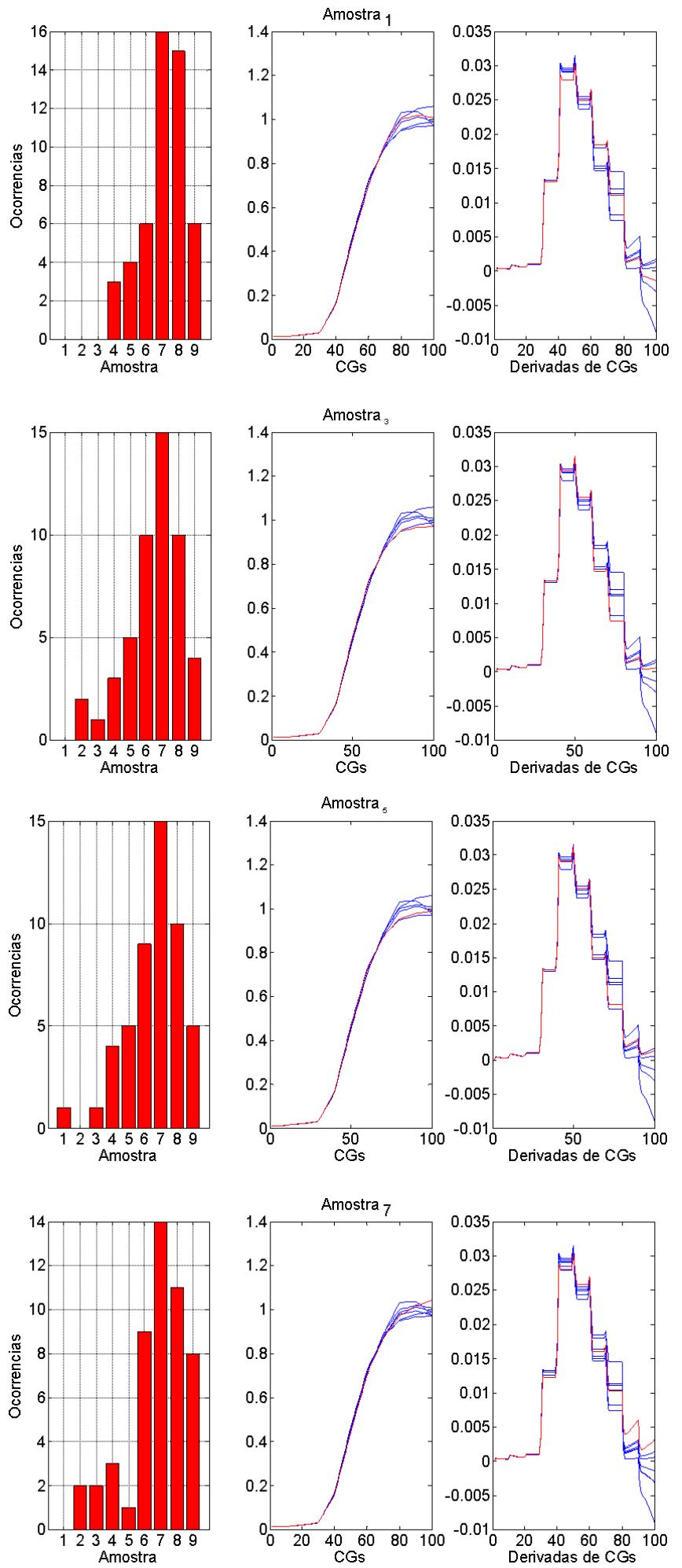

Os resultados obtidos nos traçados dos histogramas das amostras na geração das curvas CG e da sua derivada serão utilizados em um novo método para análise sensorial das amostras.

A abordagem foi acoplar o histograma à curva CG. Para cada valor representado no histograma calculou-se um novo peso, denominado "pesoh=hist/elem" em que "elem" é o número de provadores (elem $=50)$ como mostra a Eq. 1.
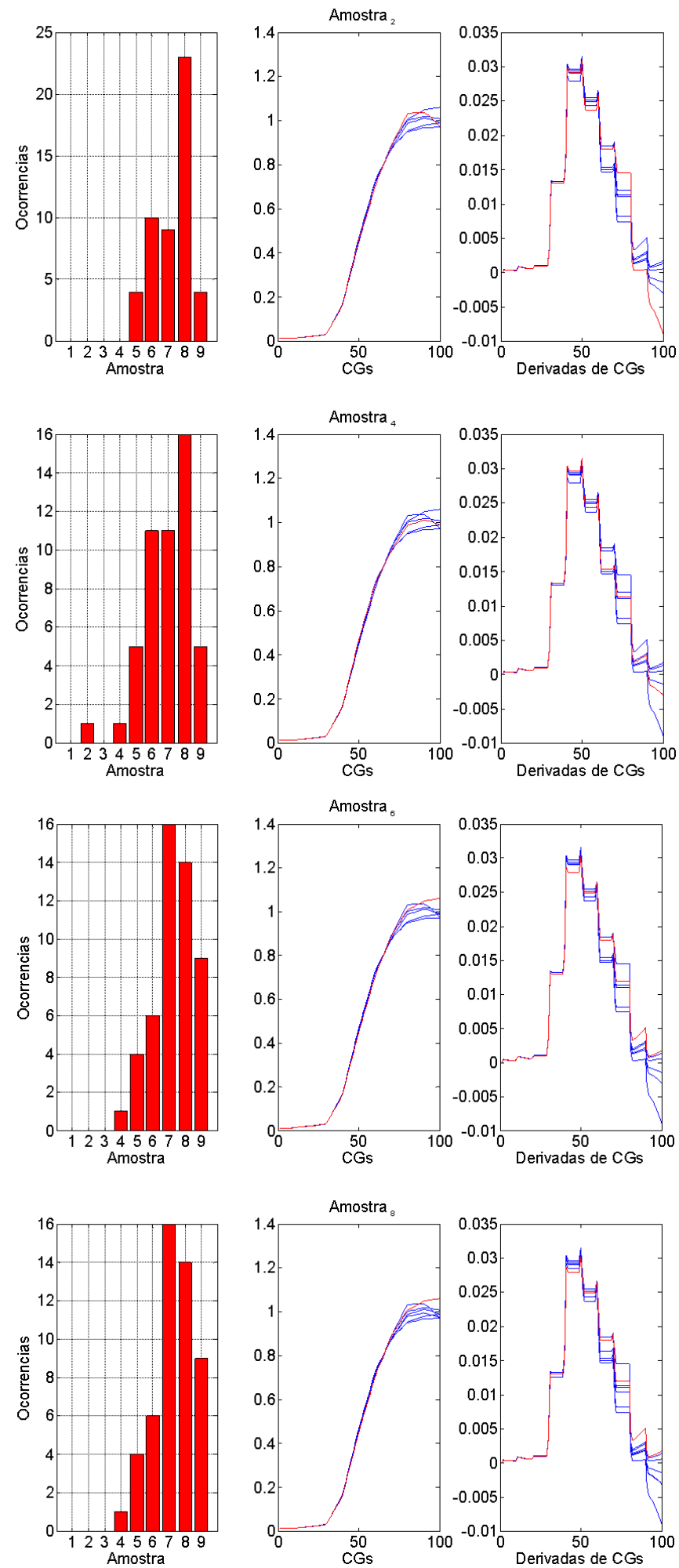

Figura 7. Resultados relativos das curvas CG das amostras (1- 8) considerando-se o cálculo do CG com histograma 
Na Figura 7 apresentam-se as curvas CG das amostras considerando-se o cálculo do CG com histograma. Observe-se que as curvas das diferentes CGs terminam em pontos diferentes porém em uma mesma área.

O valor final de $C G(X)$ das amostras analisadas foi de 1,0083 para a amostra $1 ; 0,9739$ para a amostra $2 ; 0,9719$ para a amostra 3; 0,9907 para a amostra 4; 0,9897 para a amostra 5; 0,9727 para a amostra 6; 1,0433 para a amostra 7 e 1,0616 para a amostra 8 . Analisando os valores obtido, observa-se que a amostra 8 apresentou maior valor final que as demais amostras e, após estudos comparativos com os métodos estatísticos convencionais (ANOVA), pode-se confirmar que a amostra indicada (a melhor avaliada pelos provadores) é aquela com a maior $\mathrm{CG}(\mathrm{X})$.

A amostra 8, experimento 7, equivalente ao ponto central do planejamento experimental utilizado na elaboração do pão em questão, obteve maior aceitação global pelos provadores, segundo o sistema fuzzy, uma vez que apresentou maior centro de gravidade (CG) em relação às outras concentrações analisadas; a amostra 8 obteve aproximadamente $90 \%$ de aceitação pelos provadores que atribuíram notas superiores a 5 (nem gostei/nem desgostei) na escala hedônica.Uma das exigências da avaliação sensorial é a decisão sobre a qualidade do produto, que nos testes de escala hedônica é traduzida pelo aceito.

Corroborando com os resultados apresentados, Lanzillotti \& Lanzillotti (1999) também estudaram a aplicação da lógica fuzzy na tomada de decisão em análise sensorial como alternativa para avaliar alimentos e preparações alimentares, especialmente em alimentação coletiva. Os autores aplicaram testes sensoriais com geleia de casca de banana e doce de entre casca da melancia cujos achados permitem utilizar a lógica fuzzy como alternativa às análises clássicas; enquanto a ANOVA e a MANOVA são usadas em testes para interação entre atributos, a lógica fuzzy mapeia a sensação de "prazer/ desprazer" decidindo pela convergência das funções de pertinência de forma holística.

\section{Conclusão}

A lógica fuzzy mostrou-se efetiva para análise de produtos alimentares com variáveis em suas formulações por obter resultados a partir do maior centro de gravidade (CG) tornandose uma alternativa às análises clássicas.

\section{Literatura Citada}

Azevedo, F. de L. A. A. A.; Silva, A, D. F.; Maciel, J. F.; Moreira, R. T.; Farias, L. R. G. Avaliação sensorial de pão de forma elaborado com soro de. Revista Brasileira de Produtos Agroindustriais, v.13, p.37-47, 2011.

Battochio, J. R.; Cardoso, J. M. P.; Kikuchi, M.; Macchione, M.; Modolo, J. S.; Paixão, A. L.; Pinchelli, A. M.; Silva, A. R. da. Perfil sensorial de pão de forma integral.Ciência e Tecnologia de Alimentos, v.26, p.428-432, 2006.
Brasil. Instituto Adolfo Lutz. Ministério da Saúde. Agencia Nacional de Vigilância Sanitária. Métodos físico-químicos para análise de alimentos. Brasília: Instituto Adolfo Lutz, 4.ed., 2005. 1018p.

Cavalcanti, M. T.; Bora, P. S. Análise das proteínas e estudo reológico dos isolados protéicos das amêndoas da faveleira (Cnidoscolus phyllacanthus (Mart.) Pax et K. Hoffm.) com e sem espinhos. Revista do Instituto Adolfo Lutz, v.69, p.243-251. 2010.

Cavalcanti, M. T.; Bora, P. S.; Carvajal, J. C. L. Propriedades funcionais das proteínas de amêndoas da faveleir (Cnidoscolus phyllacanthus (Mart.) Pax et K. Hoffm.) com e sem espinhos. Ciência e Tecnologia de Alimentos, v.29, p.597-602, 2009.

Cavalcanti, M. T.; Cavalcanti, J. H. F.; Silveira, D. C. da; Fernandes, A. de A.; Maracajá, P. B. Um sistema especialista com visão digital desenvolvido para selecionar e medir sementes. Revista Verde de Agroecologia e Desenvolvimento Sustentável, v.6, p.84-91, 2011a.

Cavalcanti, M. T.; Silveira, D. C. da; Florentino, E. R.; da Silva, F. L. H.; Maracajá, P. B. Caracterização biométrica e físico-química das sementes e amêndoas da faveleira (Cnidosculus phyllacanthus (Mart.) Pax. et K. Hoffm.) com e sem espinhos. Revista Verde de Agroecologia e Desenvolvimento Sustentável, v.6, p.41-45, 2011b.

Gomide, F. A. C.; Gudwin, R. R. Modelagem, controle, sistemas e lógica fuzzy.Revista da SBA Controle \& Automação, v.4, p.97-115, 1995.

Kosko, B. Additive fuzzy systems: From function approximation to learning. In: Fuzzy logic and neural network handbook. Cap.9. New York: McGraw-Hill, Inc., 1996. p.901-922.

Lanzillotti, R. S.; Lanzillotti, H. S. Análise sensorial sob o enfoque da decisão fuzzy. Revista de Nutrição, v.12, p.145157, 1999.

Liu, M.; Chen, D.; Wu, C.; Li, H. Approximation theorem of the fuzzy transform in fuzzy reasoning and its applications to de scheduling problem. Computers and Mathematics whit Aplications, v.51, p.515-526, 2006.

Mamdani, E. H.; Gaines, B. R. Fuzzy reasoning and its applications, London: Academic Press Inc., 1981. 381p.

Nolasco-Carvalho, C. C.; Franca-Rocha, W. J. S. F.; Ucha, J. M. Mapa digital de solos: Uma proposta metodológica. Revista Brasileira de Engenharia Agrícola e Ambiental, v.13, p.46-55, 2009.

Oliveira, M. A.; Montini, A. de A. Uma abordagem para análise de projetos de investimento utilizando métodos financeiros e lógica fuzzy. Revista de Administração da UNIMEP, v.7, p.134-151, 2009.

Rodrigues Júnior, F. A.; Vieira, L. B.; Queiroz, D. M.; Santos, N. T. Geração de zonas de manejo para cafeicultura empregando-se sensor SPAD e análise foliar. Revista Brasileira de Engenharia Agrícola e Ambiental, v.15, p.778787, 2011.

Zadeh, L. A. Fuzzy sets as the basis for a theory of possibility. Fuzzy Sets and Systems, v.1, p.3-28, 1978. 